\title{
Study of metabolic syndrome in postmenopausal
}

\section{women}

\author{
Alina Shri Sapkota, ${ }^{1 *}$ Alisha Sapkota, ${ }^{2}$ Kumananda Acharya, ${ }^{1}$ \\ Mithileshwer Raut, ${ }^{1}$ Bharat Jha ${ }^{1}$
}

BACKGROUND: Various studies throughout the world have documented higher prevalence of Metabolic Syndrome (MS) and CV risk factors in postmenopausal women. Abdominal obesity, a key component of metabolic syndrome is quite prevalent in South Asian women. However, studies that have investigated the effect of menopause on the health status of Nepalese women is lacking.

METHODS: This cross-sectional study was conducted in TUTH, Kathmandu Nepal. Forty five each premenopausal and postmenopausal (defined by cessation of menstruation for $\geq 12$ months) women visiting the General Health Checkup Unit were enrolled as participants. Metabolic syndrome was defined by IDF criteria. BP, height, weight and WC were measured and BMI was calculated while fasting blood samples were analyzed for serum biochemical markers: FBG, lipid profile.

RESULTS: This study found higher prevalence of MS in postmenopausal women $(57.8 \%)$ in comparison to premenopausal women (20\%). $13.3 \%$ were diabetic, $23.3 \%$ were hypertensive, $82.2 \%$ had abdominal obesity (WC>80 cm), 43.3\% were overweight (BMI $\left.25-29.99 \mathrm{Kg} / \mathrm{m}^{2}\right)$ and $13.3 \%$ were obese $(\mathrm{BMI} \geq 30$ $\mathrm{Kg} / \mathrm{m}^{2}$ ). WC, BMI and SBP were significantly higher in postmenopausal group. Among the biochemical markers, FBG, TC, LDL-C, TG were significantly higher whereas HDL-C was significantly lower in postmenopausal women than in premenopausal women.

CONCLUSION: MS was highly prevalent in postmenopausal women. This is an indication of taking necessary preventive measures so that the risk of developing diabetes and cardiovascular disease can be minimized in the postmenopausal group.

(C) 2015 Nepalese Association for Clinical Chemistry

\section{Introduction}

The presence of an adequate endogenous estrogen while the menstruation is regular in women is believed to have cardioprotective effect. Therefore, women during their fertile period of life are protected from coronary heart disease in comparison to men. However, following menopause, the ovaries permanently cease to produce estrogens and the female advantage in cardiovascular disease (CVD) present earlier would be lost. The decline in hormone level that begin few years earlier to menopause bring about various metabolic changes thereby worsening the $\mathrm{CV}$ risk profile. Metabolic disturbances including abdominal obesity, hypertension, fasting hyperglycemia, hypertriglyceridemia, low highdensity lipoprotein cholesterol (HDL-C) etc. tend to cluster together. The constellation of these metabolic disturbances is termed as metabolic syndrome [1]. The metabolic syndrome is now considered to be driving the twin global epidemics of type 2 diabetes and CVD [2].

The declining level of estrogen and alteration of its ratio with testosterone has been implicated as a causal factor for the emergence of MS at menopausal transition [3]. Alterations in lipid metabolism with estrogen deficiency are thought to be a substantial component of CVD risk in postmenopausal women [4] but there are also direct effects of estrogen deficiency on body fat distribution (central obesity), insulin action, the arterial wall, and fibrinolysis that may influence cardiovascular risk. These factors contribute to an increased prevalence of the MS in postmenopausal women compared with premenopausal women [5].

According to the national report of Central Bureau of Statistics of Nepal in 2011, total population of Nepal is $26,494,504$ out of which $13,645,463$ are women. About 1.9 million of women were 50 years of age or older. Most of these women had or shortly would have their last menstrual period, thus becoming postmenopausal. As an average Nepali woman has life expectancy of 68 years, whereas in developed countries a lifespan up to 80 years is possible, which indicates that a woman spends one-third of her life after menopause [6].Out of several health problems occurring during this period, emergence of MS is

\footnotetext{
'Department of Biochemistry, Maharajgunj Medical Campus, Institute of Medicine, Kathmandu, Nepal, ${ }^{2}$ National Public

Health Laboratory, Teku, Kathmandu
}

Correspondence to: Alina Shri Sapkota, Department of Biochemistry, Institute of Medicine.

Email: alinasapkota12@gmail.com 
of particular significance because there is substantial increase in metabolic disturbances. Globally, CVDs, often thought to be a 'male' problem, is the number one killer of women [7]. Thus, identification of postmenopa-usal women at high risk for MS has important implications for the reduction of CVD burden. CVDs are the consequences of mechanisms that are multifactorial in origin and MS includes almost all major factors for generation and progression of CVD. Therefore, the study of MS in postmenopausal women will be a single marker which can justify the multiple factors generating atherosclerosis, the cause of cardiovascular disease. International Diabetes Federation (IDF) criteria were employed for defining MS in this study.

The IDF consensus worldwide definition of the metabolic syndrome (2006) is:

Central obesity (defined as waist circumference with ethnicity-specific values*) and any two of the following [2]:

- Raised triglycerides: > $150 \mathrm{mg} / \mathrm{dL} \quad(1.7$ $\mathrm{mmol} / \mathrm{L}$ ), or specific treatment for this lipid abnormality

- Reduced HDL cholesterol: < 40 mg/dL (1.03 $\mathrm{mmol} / \mathrm{L})$ in males, $<50 \mathrm{mg} / \mathrm{dL} \quad(1.29$ $\mathrm{mmol} / \mathrm{L}$ ) in females, or specific treatment for this lipid abnormality

- Raised blood pressure (BP): systolic BP > 130 or diastolic $\mathrm{BP}>85 \mathrm{~mm} \mathrm{Hg}$, or treatment of previously diagnosed hypertension.

- Raised fasting plasma glucose (FPG): 100 $\mathrm{mg} / \mathrm{dL}(5.6 \mathrm{mmol} / \mathrm{L})$, or previously diagnosed type 2 diabetes.

If $\mathrm{FPG}$ is $>5.6 \mathrm{mmol} / \mathrm{L}$ or $100 \mathrm{mg} / \mathrm{dL}$, an oral glucose tolerance test is strongly recommended, but is not necessary to define presence of the syndrome. If $\mathrm{BMI}$ is $>30 \mathrm{~kg} / \mathrm{m}^{2}$, central obesity can be assumed and waist circumference does not need to be measure.

? To meet the criteria, waist circumference must be: for Europeans, $>94 \mathrm{~cm}$ in men and $>80 \mathrm{~cm}$ in women; and for South Asians, Chinese, and Japanese, $>90 \mathrm{~cm}$ in men and $>80 \mathrm{~cm}$ in women. For ethnic South and Central Americans, South Asian data are used, and for subSaharan Africans and Eastern Mediterranean and Middle East (Arab) populations, European data are used.

\section{Methods}

This cross-sectional study was conducted in Tribhuvan University Teaching hospital (TUTH). A total of 90 women visiting the OPD, for their general health check-up were randomly selected for the study. 45 of them were postmenopausal who had undergone natural menopause defined by cessation of menstruation for $\geq 12$ months without any other medical cause and other 45 were regularly menstruating premenopausal women. Women who were amenorrhoeic due to hysterectomy or cessation of periods other than by a natural cause, women on HRT, women having irregular menses, pregnant and lactating women were excluded from the study.

An informed consent was obtained from each participant. After that a questionnaire was completed for each participant including demographic information, menopausal status, medical history and family history. The physical examination and clinical laboratory data was also noted.

BP measurement was done using a recently calibrated aneroid sphygmomanometer with an adequate cuff size, after participant had rested for at least five minutes. Weight was taken using a platform weighing scale. Standing height measurement was done with the participants barefooted, eyes looking ahead. Body mass index (BMI) was calculated by using the formula: (weight $[\mathrm{kg}]$ ) / (Height $[\text { meter }]^{2}$ ). Using a flexible metric tape the waist circumference (in centimetres) was assessed at a point midway between the lowest rib and the iliac crest with the subject standing.

For biochemical analyses, $5 \mathrm{~mL}$ of blood was drawn after an overnight fast (8-12hours) by venipuncture. Serum samples were separated, within half an hour, by centrifugationat 1500 $3000 \mathrm{rpm}$ for $5 \mathrm{~min}$. Routine investigation was done on the same day of sample collection and included -blood glucose, TC, HDL-C, LDL-C and TG. Laboratory standard operation procedures were maintained for all laboratory analysis.

Determination of blood glucose was done by glucose oxidase peroxidise (GOD-POD) method, as described by Trinder, manufactured by Biolabo Reagents, France. Serum total cholesterol and triglyceride levels were determined enzymatically (Human, Germany). Serum HDL-C level 
was determined enzymatically after precipitation of LDLs and VLDLs with dextran sulphate $\mathrm{MgCl}_{2}$. LDL-C was calculated using the Friedewald formula, as follows.

LDL-C $(\mathrm{mmol} / \mathrm{L})=$ Total cholesterol (Triglyceride/2.2 + HDL cholesterol)

When triglyceride concentration exceeded $4.5 \mathrm{mmol} / \mathrm{L}$, LDL-C was estimated by direct homogenous method (Biolabo, France). All the biochemical tests were performed by the fully automated chemistry analyzer, BT3000, Italy. Metabolic syndrome was defined by IDF criteria.

The data were entered in Microsoft Excel program (Microsoft office 2007). Statistical analyses were done by SPSS 20.0 version (Statistical Package for Social Science for Windows version; SPSS Inc., Chicago, IL). Mean

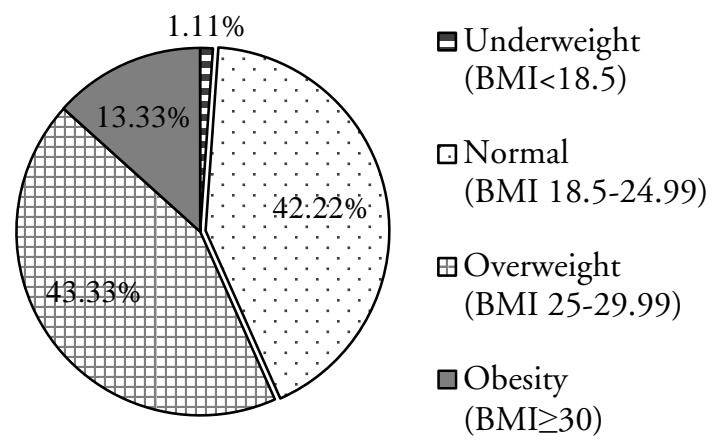

Fig. 1. Distribution of the study population based on BMI $(n=90)$.

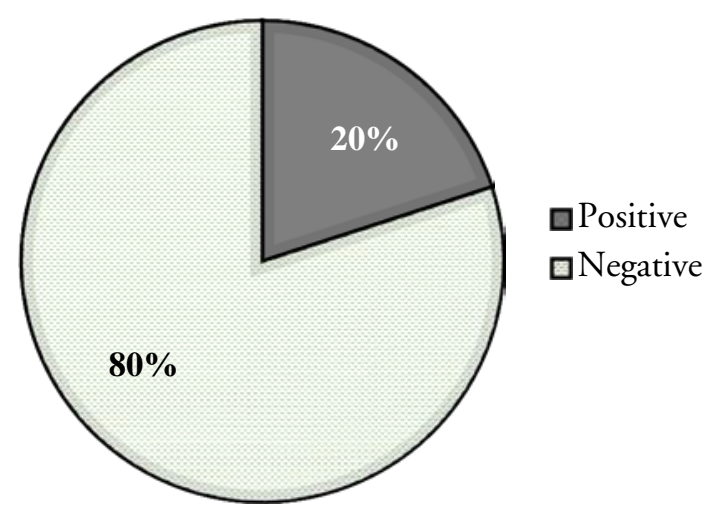

Fig. 3. Prevalence of MS in premenopausal $(n=45)$. comparison was done by t-tests.

\section{Results}

Out of total 90 participants in this study, 45 were regularly menstruating premenopausal women of age group 20-40 years. Remaining 45 were post-menopausal women who had cessation of menstruation at least 12 months earlier and they were of age group 45-70. Mean age of menopause was 48.9 years. Out of 90 participants $13.3 \%$ had the history of diabetes and $23.3 \%$ had the history of hypertension.

\section{Discussion}

Evidence of clustering of various cardiometabolic derangements and CVD risk factors has been found in postmenopausal women in this study.

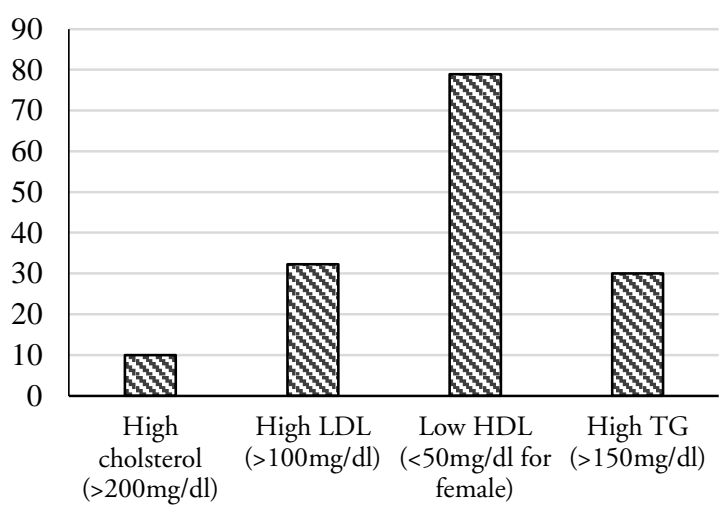

Fig. 2. Prevalence of Dyslipidemia $(n=90)$.

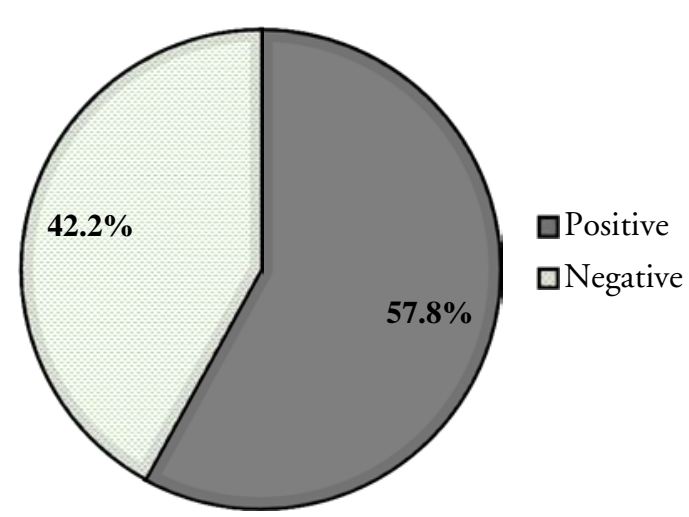

Fig. 4. Prevalence of MS in postmenopausal women $(n=45)$. 
Table 1. Comparison of Anthropometric and biochemical parameters between pre and postmenopausal women

\begin{tabular}{lccc}
\hline Parameter & $\begin{array}{c}\text { Premenopausal } \\
(\mathrm{n}=45) \\
\text { Mean } \pm \text { SD }\end{array}$ & $\begin{array}{c}\text { Postmenopausal } \\
(\mathbf{n}=45) \\
\text { Mean } \pm \text { SD }\end{array}$ & p value \\
\hline Age $($ Years $)$ & $33.8 \pm 5.7$ & $57.9 \pm 5.7$ & $<0.001$ \\
SBP $(\mathrm{mmHg})$ & $112.5 \pm 13.7$ & $122.6 \pm 12.1$ & $<0.001$ \\
DBP $(\mathrm{mmHg})$ & $76.5 \pm 9.8$ & $79.4 \pm 8.7$ & 0.145 \\
Height $(\mathrm{cm})$ & $154.8 \pm 4.3$ & $150.8 \pm 5.5$ & $<0.001$ \\
Weight $(\mathrm{Kg})$ & $60.2 \pm 7.8$ & $61.1 \pm 10.1$ & 0.589 \\
BMI $\left(\mathrm{Kg} / \mathrm{m}^{2}\right)$ & $25.1 \pm 3.4$ & $26.9 \pm 3.8$ & 0.028 \\
Waist Circumference $(\mathrm{cm})$ & $84.8 \pm 9.6$ & $94.3 \pm 10.1$ & $<0.001$ \\
Glucose $(\mathrm{mmol} / \mathrm{L})$ & $4.6 \pm 0.6$ & $5.3 \pm 1.2$ & 0.002 \\
Total cholesterol $(\mathrm{mmol} / \mathrm{L})$ & $3.85 \pm 0.7$ & $4.21 \pm 0.9$ & 0.028 \\
Triglygeride (mmol/L) & $1.33 \pm 0.6$ & $1.69 \pm 0.6$ & 0.006 \\
HDL-C $(\mathrm{mmol} / \mathrm{L})$ & $1.12 \pm 0.2$ & $1.01 \pm 0.2$ & 0.021 \\
LDL-C $(\mathrm{mmol} / \mathrm{L})$ & $2.12 \pm 0.6$ & $2.43 \pm 0.7$ & 0.028
\end{tabular}

P-value $<0.05$ is statistically significant

The prevalence of MS was found to be $57.8 \%$ in postmenopausal and $20 \%$ in premenopausal women using the IDF criteria. Our findings were consistent with many of previous studies [8-13], where postmenopausal women were found to be at higher risk of MS than premenopausal women. The prevalence of MS has greatly varied across different studies. According to a study of Western India the prevalence of MS was 45\% among premenopausal women, whereas it was $55 \%$ among postmenopausal women [11]. Eshtiaghiet. al., showed a prevalence of $53.5 \%$ MS in postmenopausal Iranian women, on the other hand it was only $18 \%$ in pre-menopausal women [10]. Differences in socio-environmental and genetic factors, lifestyles, type of menopause (natural/ surgical), time since menopause and criteria used for defining MS could be some of the reasons for this variability.

In the present study, significant differences in waist circumference and BMI among pre and post-menopausal women have been found and this is similar to some other studies [8, 14]. $82.2 \%$ of postmenopausal subjects are having abdominal obesity as defined by waist circumference $>80 \mathrm{~cm}$. Cross-sectional [15] and longitudinal studies [16] have shown that the menopausal transition is associated with a preferential increase in abdominal adiposity, independent of the effect of age and total body adiposity. In the present study abdominal obesity was also observed in $72.5 \%$ of premenopausal subjects. This could be due to various factors including physical inactivity, dietary habits, socioeconomic or genetic factor. This implies that abdominal obesity is the leading factor for metabolic syndrome. Current evidence implies that multiple risk factors for CVD emerge in the postmenopausal period, but features of the MS may be present even before menopause [17]. Moreover, South Asian Indians, in general, are prone to have MS at a younger age and have severe morbidity and mortality consequences as compared to Caucasians $[18,19]$.

While classifying the study population based on their BMI, only $1.1 \%$ were underweight, $42.2 \%$ had normal BMI, whereas, $43.3 \%$ were overweight and $13.3 \%$ were obese respectively. Altogether, $56.6 \%$ had higher than the normal BMI $\left(\geq 25 \mathrm{Kg} / \mathrm{m}^{2}\right)$ and $82.2 \%$ of the women had raised WC $(>80 \mathrm{~cm})$. This is an alarming sign which indicates that lifestyle modification and dietary habit change is essential to control and prevent obesity and the consequences related to it.

In present study, a significantly higher level of metabolic risk factors including fasting blood glucose, total cholesterol, triglyceride and LDL-C were observed among post-menopausal women 
than pre-menopausal women. HDL-C was also found to be significantly lower in the postmenopausal group. In agreement with the results of our study, many previous studies have reported higher prevalence of hypercholesterolemia $[13,14,20,21]$, hypertriglyceridemia [9, 13, 20-22], high LDL-C [8, 13, 20, 21], low HDL-C [20, 21] and elevated fasting blood glucose $[13,22,23]$ among post postmenopausal women than pre-menopausal women. A high amount of abdominal fat is associated with increased insulin resistance, free fatty acid (FFA) levels, and decreased adiponectin. These factors contribute to increased secretion of apolipoprotein B (apo-B)-containing particles, leading to hypertriglyceridemia and increased hepatic lipase (HL) activity resulting in a predominance of small dense LDL particles and a reduction in large antiatherogenic HDL2 particles. A similar pattern of lipid abnormalities emerges with menopause [24]. Insulin resistance, with inadequate compensatory hyperinsulinemia, diminishes the normal suppression of FFA arising from adipose tissue by insulin. The increased levels of FFA may impair peripheral glucose uptake, increase hepatic gluconeogenesis, and reduce hepatic clearance of insulin [25].

In present study, a significantly higher level of systolic blood pressure was observed among postmenopausal women than pre-menopausal women $(p=<0.001)$ whereas the mean diastolic blood pressure though elevated in post-menopausal group was not significantly different from the pre-menopausal group. Previous studies have reported higher prevalence of systolic blood pressure [13]. The decline in the oestrogen/androgen ratio dilutes the vasorelaxant effects of oestrogens on the vessel wall and promotes the production of vasoconstrictive factors such as endothelin [26]. Both male and female sex steroids have a regulating effect on the renin-angiotensin system (RAS) and affect angiotensinogen production and sodium metabolism. The decline in oestrogen levels around menopause causes an up regulation of the RAS with an increase in plasma renin activity [27]. Hypertension often clusters with other risk factors such as overweight, elevated insulin resistance, diabetes and lipid abnormalities.

\section{Conclusion}

MS was found high in postmenopausal women than in premenopausal women. Prevalence of MS was $57.8 \%$ in postmenopausal women and $20 \%$ in premenopausal group. Such a high prevalence of MS in postmenopausal group is an alarming sign. Prevention through changes in lifestyle, or early detection and treatment of elevated fasting blood glucose, hypertension, and hyperlipidemia are necessary for prevention of cardiovascular diseases in Nepalese women reaching menopause. Health professionals should consider the postmenopausal women as a major target group for prevention of MS, which is an underlying condition of many non-communicable diseases. 


\section{References}

1. Alberti KG, Zimmet PZ. Definition, diagnosis and classification of diabetes mellitus and its complications. Part 1: diagnosis and classification of diabetes mellitus provisional report of a WHO consultation. Diabetic medicine : a journal of the British Diabetic Association. 1998;15(7):539-53.

2. Intenational Diabetes Federation. The IDF Consensus Worldwide Definition of the Metabolic Syndrome. Brussels, Belgium: Intenational Diabetes Federation 2006.

3. Mesch VR, Boero LE, Siseles NO, Royer M, Prada M, Sayegh F, et al. Metabolic syndrome throughout the menopausal transition: influence of age and menopausal status. Climacteric. 2006;9(1):40-8.

4. Kannel WB, Wilson PW. Risk factors that attenuate the female coronary disease advantage. Archives of internal medicine. 1995;155(1):57-61.

5. Park YW, Zhu S, Palaniappan L, Heshka S, Carnethon MR, Heymsfield SB. The metabolic syndrome: prevalence and associated risk factor findings in the US population from the Third National Health and Nutrition Examination Survey, 1988-1994. Archives of internal medicine. 2003;163(4):42736.

6. Government of Nepal NPncS, Central Bureau of Statistics. National Population and Housing Census 2011

7. WHO. Women's health; Fact sheet No334 2013. Available from: http://www.who.int/mediacentre/fac tsheets/fs334/en/. Accessed on: 13 Jan 2015

8. Lin WY, Yang WS, Lee LT, Chen CY, Liu CS, Lin CC, et al. Insulin resistance, obesity, and metabolic syndrome among non-diabetic preand post-menopausal women in North Taiwan. Int $\mathrm{J}$ obesity 2006;30(6):912-7.

9. Chhabra N, Sodhi K, Kukreja S, Chhabra S, Vijayasarathy S, Chhabra $S$, et al. Central obesity and prevalence of metabolic syndrome in post-menopausal women. 2014.
10. Eshtiaghi R, Esteghamati A, Nakhjavani M. Menopause is an independent predictor of metabolic syndrome in Iranian women. Maturitas. 2010;65(3):262-6.

11. Pandey S, Srinivas $M$, Agashe $S$, Joshi J, Galvankar P, Prakasam CP, et al. Menopause and metabolic syndrome: A study of 498 urban women from western India. Journal of mid-life health. 2010;1(2):63-9.

12. Jesmin $S$, Islam A, Akter $S$, Islam M, Sultana S, Yamaguchi N, et al. Metabolic syndrome among pre- and post-menopausal rural women in Bangladesh: result from a population-based study. BMC research notes. 2013;6(1):1-7.

13. Kim HM, Park J, Ryu SY, Kim J. The effect of menopause on the metabolic syndrome among Korean women: the Korean National Health and Nutrition Examination Survey, 2001. Diabetes care. 2007;30(3):701-6

14. Ebrahimpour P, Fakhrzadeh $H$, Heshmat R, Ghodsi M, Bandarian F, Larijani B. Metabolic syndrome and menopause: A population-based study. Diabetes \& Metabolic Syndrome: Clinical Research \& Reviews. 2010;4(1):5-9.

15. Zamboni M, Armellini F, Milani MP, De Marchi M, Todesco T, Robbi R, et al. Body fat distribution in pre- and post-menopausal women: metabolic and anthropometric variables and their inter-relationships. International journal of obesity and related metabolic disorders : journal of the International Association for the Study of Obesity. 1992;16(7):495504.

16. Bjorkelund C, Lissner L, Andersson S, Lapidus L, Bengtsson C. Reproductive history in relation to relative weight and fat distribution. International journal of obesity and related metabolic disorders. 1996;20(3):213-9.

17. Carr MC. The emergence of the metabolic syndrome with menopause. The Journal of clinical endocrinology and metabolism. 2003;88(6):2404-11.
18. Misra A, Khurana L. Obesity and the metabolic syndrome in developing countries. The Journal of clinical endocrinology and metabolism. 2008;93(11 Suppl 1):S9-30.

19. Pan WH, Yeh WT, Weng LC. Epidemiology of metabolic syndrome in Asia. Asia Pacific journal of clinical nutrition. 2008;17 Suppl 1:37-42.

20. Bade G, Shah S, Nahar P, Vaidya S. Effect of menopause on lipid profile in relation to body mass indexJanuary 1, 2014. 20-4 p.

21. Reddy Kilim S, Chandala SR. A comparative study of lipid profile and oestradiol in pre- and postmenopausal women. Journal of clinical and diagnostic research : JCDR. 2013;7(8):1596-8.

22. Kow Nanse Arthur F, AduFrimpong M, Osei-Yeboah J, Obu Mensah F, Owusu L. The prevalence of metabolic syndrome and its predominant components among pre-and postmenopausal Ghanaian women. BMC research notes. 2013;6(1):446.

23. Jouyandeh Z, Nayebzadeh F, Qorbani M, Asadi M. Metabolic syndrome and menopause. Journal of diabetes and metabolic disorders. 2013;12(1):1.

24. Carr MC. The Emergence of the Metabolic Syndrome with Menopause. The Journal of Clinical Endocrinology \& Metabolism. 2003;88(6):2404-11.

25. Despres JP. Abdominal obesity as important component of insulinresistance syndrome. Nutrition (Burbank, Los Angeles County, Calif). 1993;9(5):452-9.

26. Reckelhoff JF, Fortepiani LA. Novel mechanisms responsible for postmenopausal hypertension. Hypertension. 2004;43(5):918-23.

27. Schunkert H, Danser AH, Hense HW, Derkx FH, Kurzinger S, Riegger GA. Effects of estrogen replacement therapy on the reninangiotensin system in postmenopausal women. Circulation. 1997;95(1):39-45. 